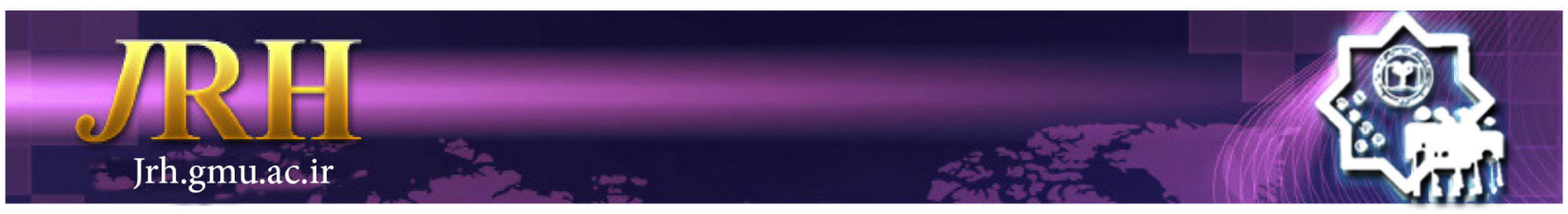

\title{
The sleep quality and prevalence of sleep disorders in adolescents
}

Somayeh Safarzade ${ }^{1}$, Hamidreza Tohidinik ${ }^{2}$

\author{
Journal of Research \& Health \\ Social Development \& Health Promotion \\ Research Center \\ Vol. 9, No. 6, Nov \& Dec 2019 \\ Pages: 471 - 479 \\ DOI: $10.32598 / j r h .9 .6 .471$ \\ Original Article
}

1. Correspondence to: Department of Psychiatry, School of Medicine, Gonabad University of Medical Sciences, Gonabad, Iran

Email: Somayeh.safarzade@yahoo.com

2. Department of Public Health, School of Health, Gonabad University of Medical Sciences, Gonabad, Iran

Received: 8 Jan 2015

Accepted: 19 May 2015

How to cite this article: Safarzade S, Tohidinik H. Investigating sleep quality and epidemiology of sleep disorders in adolescents. J Research \& Health2019; 9(6): $471-479$.

\begin{abstract}
Sleep has a key role in the development of adolescents. Sleep is effective not only in physical growth, but also in behavior, emotion, cognition, attention, and school performance of adolescents. The purpose of the present study was to investigate the quality of sleep and the prevalence of sleep disorders in adolescents of Gonabad city. The statistical sample consisted of 1153 adolescents studying in the secondary high schools of Gonabad city. A demographic questionnaire, the pittsburgh sleep quality scale, and global sleep assessment questionnaire were used to collect data. $75.3 \%$ of the adolescents had low and inappropriate sleep quality. In addition, disturbances in beginning of sleep (16.7\%), Disturbances in maintaining sleep $(11.5 \%)$, daytime fatigue $(31.6 \%)$, daytime sleepiness (24.7\%), sleep disorder due to daily intense activity (27.8\%), Breathing pause $(10.2 \%)$, snoring $(9.4 \%)$, restless leg syndrome $(16.9 \%)$, periodic movements limb (11.7\%), sleepwalking disorder (11\%), having nightmare ( $8.5 \%)$, secondary insomnia (18.2\%), feelings of anxiety and worry (26.4\%), and Disturbances in daily work (26\%) were the other obtained results. According to the poor quality of sleep and prevalence of sleep disorders in them, it is essential to respect the causes of the issue to reduce the negative consequences.
\end{abstract}

Keywords: Adolescent, Prevalence, Sleep

\section{Introduction}

Sleep is a natural phenomenon considered as one of the basic physiological needs of humans. Sleep is a dynamic and highly organized biological process encompassing one-third of the human life [1]. Sleep is a regular, repeating, and easily reversible state of the organism, identified with relative motion and high reaction threshold to exterior stimuli compared to individuals' awaken state $[2,3]$. Sleep control is considered as the most important physiological cycle of the body and a critical, necessary, and important component of the clinical work of psychiatrists and psychologists as a disturbance in the process of sleep can be one of the early signs of sleep disorder adversely affecting a person's quality of life [4]. Sleep-related complaints are the second leading cause for people's reference to health care centers and a major problem in today's societies. Nevertheless, studies examining this disorder among different populations have reported different 
rates [5]. The range of this type of disorders in different studies conducted among the general population is from 5 to $50 \%$ [6] and in the primary care centers it is in a similar range from $10 \%$ and $50 \%$ [7].

Adolescence is known as a high-risk factor for sleep disorders. Sleep disorders in adolescents are usual and healthcare professionals face many adolescents suffering from some kinds of sleep disorders [8]. Adolescents have many biological changes, such as modifying sleep, awake, and sleep patterns settings (reduced delta sleep, reduced Rapid Eye Movement (REM) Sleep) [9]. Precise methodological studies have stressed the need of adolescents due to biological, psychological, and cultural force storms [10]. During adolescence, many changes occur in adolescent brain development. Adolescent's sleep has a great role in the healthy brain development. Adolescents need more sleep compared to pre-puberty time, but there is much turmoil in their sleep and waking cycles [11].

The reason for sleep disorders in adolescents is not clear. Sometimes, one reason is involved and sometimes a combination of several factors is responsible [12]. The role of demographic, social, and psychological factors has been proven in sleep disorders in children and adolescents. Stressors in a classroom environment, like exams or conflicts with parents, are known as effective factors in the occurrence of sleep disorders in adolescents [13,10]. Sleep health in adolescents is very important for achieving all-round growth. Sleep is effective not only in the physical growth, but also in behavior, emotions, cognitive function, school performance, and attention of adolescents [14]. Sleep disorders in adolescents cause inability to rise during school time, moodiness during the day, irritability $[15,16]$, lack of concentration [4], destruction of abstract thinking, creativity, learning [9], significant behavioral problems [15], and disorders in motor skills and reaction time [15]. The abuse of drugs [17] and absence from the workplace or school are very common among these adolescents [9]. Sleep disorders in adolescents are sometimes so serious that they cause cardiovascular disease and unpleasant metabolic effects [6].

When an adolescent enters school, some changes occur in her/his sleep pattern making some changes in her/his sleep and waking cycle. The successful implementation of these changes depends on the quality of sleep, sleeping and awakening, and behaviors during adolescence. In adolescents who do not fit themselves with the school's conditions, a combination of late-night activities and school requirements will lead to a coherent disturbance in their nightly cycles [9]. Great attention has been paid to the issue of sleep, its length, and quality in adolescents and young people in recent years. In a study $37.2 \%$ of adolescents had sleep disorders [5].

The prevalence of sleep disorders in children and adolescents in Iran is estimated to be between $25 \%$ and $30 \%$ [14]. Some studies have shown that the prevalence of sleep disorders is higher in girl adolescents than in boys, but this finding is not reliable, and other scholars have found this result dependent on racial and false perceptions. In a study, it was stressed that the presence of disturbances in the sleep and awakening cycle of adolescents causes chronic and severe illnesses [18].

Thus, it is very important to identify, evaluate, and treatit. Furthermore, despite the unpleasant effects of sleep disorders on adolescents, it is usually ignored not only in Iran but also in many countries such as England and France. Sometimes, parents do not consider seriously their adolescents' peculiar behaviors and sleep disorders but consider them periodical and unimportant. However, not considering a proper treatment for an adolescent with sleep disorders will make it sustainable in her/his youth. Sleeping hygiene education is essential for the parents of the current adolescents and future parents. In the present study, we decided to study the quality of sleep and the prevalence of sleep disorders in girl and boy adolescents. It is hoped that using the results of this study, we can identify sleep disorder in adolescents besides providing therapeutic and rehabilitation programs for reducing 
these disturbances and undesirable effects on lifestyle activities.

\section{Method}

In this study, we examined male and female students from second-grade secondary schools in Gonabad in the school year. The participants were selected according to stratified random sampling and 1200 individuals were selected to reach the research objectives. Considering the research objectives and previous studies, the sample size was determined as 995 based on the prevalence of $37 \%$, the accuracy of 0.03 , and $95 \%$ confidence level [19]. As a stratified random sampling was used in the schools, 1200 people were studied including 600 girls and 600 boys. Then, 20 secondary high schools (10 high schools for girls and 10 for boys) were randomly selected and 60 participants were examined from each. In the end, 23 girls and 15 boys were excluded from the study given their incomplete questionnaires, leaving 577 participants in the girl's group and 576 participants in the boy's group. The age range of the participants was between 14 and 19 years. Giving informed consent, being a student in the study period, and having physical and mental health were among the inclusion criteria in this study. The tools used in this study were a demographic checklist, the pittsburgh sleep quality scale, and the general sleep evaluation.

In this study, pittsburgh sleep quality scale was used to measure the quality of sleep in adolescents along with the general sleep measurement questionnaire that has been widely used in studies to determine sleep disorders precisely and as a very useful tool in this studies. Pittsburgh sleep quality scale: This questionnaire is a standard 18-question questionnaire examining the attitude of patients toward sleep quality over the past four weeks. The questionnaire has seven separate subscales: subjective sleep quality, sleep latency, sleep duration, sleep efficiency, daytime dysfunction, sleep disturbance, and use of sleeping medications. The scores of each subscale of the questionnaire range between 0 and 3 . The scores $0,1,2$, and 3 in each subscale show the natural state, a mild, moderate, and severe problem, respectively. The sum of the scores of all seven subscales will determine the total score of the questionnaire, which will be between 0 and 21 . The score between 0 and 5 indicates a good quality for sleep while a score of 5 or more than 5 indicates a poor sleep quality. The reliability of this tool has been reported to be high and its Cronbach's alpha coefficient has been obtained from 0.77 to 0.81 [4].

General sleep measurement questionnaire: This is a simple, reliable, and valid instrument for measuring sleep disorders developed by Roth and Calgiois. This inventory involves sleep disorders such as over-sleep, respiratory interruption in sleep, restless leg syndrome, sleepwalking, nightmare, the intermittent motion of the legs, secondary insomnia, and daily dysfunction due to sleep disturbance, anxiety, and depression [20]. This questionnaire has 11 questions that examine sleep disorders on a Likert scale of never, sometimes, usually, and always. The answers "always" and "usually" show a sleep disorder and the answers "sometimes" and "never" indicate the lack of a disorder. The score for each question is between 0 and 3 . The testretest reliability of this tool was obtained between 0.51 and 0.92 . The concurrent validity of this questionnaire was highly desirable in the research of Roth et al [20]. Cronbach's alpha coefficient of this questionnaire was 0.87 in Iran and its test-retest reliability was 0.86 with a four-week interval, which shows the desired reliability of this questionnaire in Iran [21]. Data were analyzed using SPSS-16 and by descriptive statistics and independent t-test, Mann-Whitney U, and Chi-square tests. Significance level at $<0.05$.

\section{Results}

The mean and standard deviation of age were 16.14 and 1.20 , respectively. The mean and standard deviation of the scores for the participants in the study during bedtime, measured by the first question of the pittsburgh sleep quality scale, were 9.19 and 3.81, 
respectively. The mean and standard deviations of the scores for the girls participating in the study were 0.92 and 3.28, and for boys, 55.8 and 18.4, respectively. The significance level in the t-test analysis of the two independent groups was 0.0001 , which was less than 0.05 showing a significant difference between the two groups of girls and boys in the hour of going to bed, and girls went to bed later than boys did $(\mathrm{p}<0.05)$. The average sleep time after bedtime was 21.41 and the standard deviation was 18.81 . The mean for girls was 22.33 with a standard deviation of 21.53 while for the sample of boys participating in the research, the mean and standard deviation were 20.49 and 15.59 , respectively. The results of t-test showed no significant differences between the two groups in the mean sleep time ( $p>0.05$ ). The mean and standard deviation of the wake-up time of the whole samples were 6.51 and 1.21, respectively. The mean and standard deviation for girls were 6.28 and 1.29, respectively, and for boys were 6.74 and 1.15 , respectively. The significance level was 0.0001 , which indicates a significant difference in the average wakeup time between the two groups with girls waking up earlier $(p<0.05)$. The mean and standard deviation of time spent in the bed for 1153 participants in the study were 6.89 and 2.53 , respectively, due to the time spent in bed going to sleep from waking up hours. The mean and standard deviation of the girls were 6.58 and 2.36, respectively, and for the boys were 7.20 and 2.65 , respectively. The significance level in the t-test for the two independent groups was $p<0.0001$ that showed a significant difference in bedtime between the two groups of boys and girls $(p<0.05)$. In Table 1 , the mean and standard deviation of the scores of the research participants based on the seven subscales of the pittsburg questionnaire and the overall score of sleep quality are presented.

\begin{tabular}{lcc}
$\begin{array}{l}\text { Table } 1 \text { The mean and standard deviation of the scores of the participants } \\
\text { based on the subscales and the whole scale of pitsburgh sleep quality }\end{array}$ \\
\hline Scale & Mean & $\begin{array}{c}\text { Standard } \\
\text { deviation }\end{array}$ \\
\hline Subjective sleep quality & 1.06 & 0.83 \\
Sleep disturbance & 1.08 & 0.45 \\
Use of sleeping medications & 0.16 & 0.49 \\
Sleep duration & 1.07 & 1.05 \\
Daytime dysfunction & 0.97 & 0.77 \\
Sleep latency & 1.06 & 0.85 \\
Sleep efficiency & 2.23 & 1.23 \\
Overall score of sleep quality & 7.68 & 3.12 \\
\hline
\end{tabular}

Mann-Whitney U test was used to determine whether there is a significant difference in the overall score of sleep quality between boys and girls. In analyzing the difference between girls $(\mathrm{Md}=8, \mathrm{n}=577)$ and boys $(\mathrm{Md}=8, \mathrm{n}=576)$, the overall score of sleep quality was significantly different $(\mathrm{U}=150723, \quad \mathrm{Z}=-2.74, \quad \mathrm{p}=0.006)$. There was a statistically significant difference in the overall score of sleep quality $(p<0.05)$. The average score in girls was 550.22 and in boys was 83.603 . Getting a score of zero in each subscale of pittsburgh sleep quality scale suggests a natural status, obtaining a score of one shows the existence of a mild problem, a score of two shows a moderate problem, and finally obtaining a score of 3 indicates a severe problem in that subscale. Table 2 shows the frequency and percentage of participants in the research based on the scores for each subscale.

Considering the general score of adolescents, the sample of the present study included 868 people, comprising $75.3 \%$ of the sample size, that their nightly sleep cycle had a poor quality. In contrast, 285 (24.7\%) had a good nighttime sleep quality. Chi-square test was used to examine whether the proportion of females with undesirable sleep has a significant 
difference with that of boys with poor sleep

quality (Table 3).

Table 2 Frequency and percentage of participants in the research in acquired scores based on each subscale

\begin{tabular}{|c|c|c|c|c|c|c|c|c|}
\hline \multirow{2}{*}{ Scale } & \multicolumn{2}{|c|}{ Zero } & \multicolumn{2}{|c|}{ One } & \multicolumn{2}{|c|}{ Two } & \multicolumn{2}{|c|}{ Three } \\
\hline & Frequency & Percent & Frequency & Percent & Frequency & Percent & Frequency & Percent \\
\hline Subjective sleep quality & 293 & 25.4 & 556 & 48.2 & 236 & 20.5 & 68 & 5.9 \\
\hline Sleep disturbance & 66 & 5.7 & 929 & 80.6 & 151 & 13.1 & 7 & 6 \\
\hline Use of sleeping medications & 1010 & 87.6 & 107 & 9.3 & 24 & 2.1 & 12 & 1 \\
\hline Sleep duration & 417 & 36.2 & 415 & 36 & 135 & 11.7 & 186 & 16.1 \\
\hline Daytime dysfunction & 329 & 28.5 & 555 & 48.1 & 238 & 20.6 & 31 & 2.7 \\
\hline Sleep latency & 314 & 27.2 & 511 & 44.3 & 262 & 22.7 & 66 & 5.7 \\
\hline Sleep efficiency & 232 & 20.1 & 62 & 5.4 & 58 & 5 & 801 & 69.5 \\
\hline
\end{tabular}

Table 3 Frequency and percentage of the prevalence of desirable or undesirable sleep quality in subjects of research based on gender

\begin{tabular}{lcccccc}
\hline \multirow{2}{*}{ Gender } & \multicolumn{2}{c}{ Desirable sleep quality } & \multicolumn{2}{c}{ Undesirable sleep quality } & \multirow{2}{*}{$\left(\mathrm{X}^{2}\right)$} & $\mathrm{p}$-value \\
\cline { 2 - 5 } & Frequency & Percentage & Frequency & Percentage & & \\
\hline Girls & 159 & 27.6 & 418 & 72.4 & 4.96 & 0.03 \\
Boys & 126 & 21.9 & 450 & 78.1 & & \\
Total & 285 & 24.7 & 868 & 75.3 & & \\
\hline
\end{tabular}

As shown in Table 3, the significance level in the Chi-square test was 0.03 , which indicates a significant difference in the quality of sleep between the two groups, with the boys having a more unfavorable quality of the overnight sleep cycle $(\mathrm{p}<0.05)$.
Regarding the way that the participants responded to the questionnaire of general sleep measurement, it was revealed how many people in each disorder have it. Table 4 presents the frequency distribution of the participants participants in sleep disorders.

Table 4 Frequency distribution of sleep disorders in participants of the research

\begin{tabular}{lcc}
\hline Type of disorder & Frequency & Percent \\
\hline Disturbances at the beginning of sleep & 192 & 16.7 \\
Disturbances in maintaining sleep & 133 & 11.5 \\
Daytime sleepiness & 285 & 24.7 \\
Sleep disorder due to daily intense & 321 & 27.8 \\
activity & 364 & 31.6 \\
Daytime fatigue & 303 & 26.3 \\
Disruptions in daily work & 118 & 10.2 \\
Breathing pause & 108 & 9.4 \\
Snoring & 195 & 16.9 \\
Restless leg syndrome & 135 & 11.7 \\
Periodic movements limb & 127 & 11 \\
Sleepwalking disorder & 98 & 8.5 \\
Nightmares & 304 & 26.4 \\
Feelings of anxiety and worry & 210 & 18.2 \\
Secondary insomnia & &
\end{tabular}

\section{Discussion}

The most important finding of the present study was that, unfortunately, $75.3 \%$ of the adolescents participating in the study had an unfavorable state in the sleep quality. In the study, 11 to $47 \%$ of the adolescents had a poor sleep quality [22]. In the study by Johnson and Breslau, $6 \%$ of adolescents had a poor quality of sleep [23]. In a study in Australia, it was 
found that $37.6 \%$ of adolescents complained about their sleep, awakening, and sleep quality [24]. The value achieved in this study is greater than the reported values in some studies. In explaining this difference, it is very important to note that a distinct culture may lead to very different results from another culture. For example, in a study by Cornelius, Spanish and African adolescents had more disorders in their sleep compared to American and European adolescents [25].

The results of the present study showed a significant difference between girls and boys in the total score of sleep quality so that the overall score of boys in the category of sleep quality was higher than that of girls. The results of this study are consistent with the results of Fernando et al [5]. In that study, the researchers found sleep disorders and poor sleep quality in boys as $42.7 \%$ and in girls as $33 \%$, which is consistent with the results of the present study. There were gender differences in sleep disorders in the study by Meltzer et al. in 2010 so that the prevalence of poor quality of sleep for boys was $6.3 \%$ and for girls was $5.8 \%$. The results of that study are in line with the findings of the present study [26]. In the study by Kaneita et al, it was found that poor sleep quality and sleep disorders were more prevalent in girls than in boys, which is inconsistent with the results of the present study [10].

The results of this study showed that $1 \%$ of the adolescents had very frequent use of sleeping pills. In the study by Mousavi et al., the use of sleeping medications in the adolescent and youth groups was $1.2 \%$, which is somehow the same as in the present study [27]. In the study by Modarresi et al., the prevalence of sleeping medication consumption was $1.6 \%$, which is approximately the same as the prevalence in the present study [28]. In explaining this observation, it is noteworthy that insomnia that sometimes exacerbates the consumption of sleeping medications may be due to anxiety, depression, or other mental and physical problems, familial, environmental, and economic conditions and the use of these drugs may reduce the problem of insomnia in the first few weeks, making it easy for them to sleep, but in the long-run, it will be addictive and the adolescent becomes accustomed to it. Moreover, not only will his insomnia not be solved, but also progressively, the negative effects on the body and soul of the adolescent will show up. Thus, according to the results of this study, although a small percentage of adolescents had severe sleep deprivation, even this small percentage needs attention, so that the main cause of their sleeplessness is identified, and the solutions and interventions for optimal psychotherapy and psychiatry will be taken to reduce the problem. Moreover, the results showed that $16.1 \%$ of the adolescents had a low sleep time, and $5.7 \%$ had a severe delay in sleeping. The results of a study in the US showed that sleep disorders in adolescents and young people have grown more in recent years, so that the proportion of young people who sleep between 8.5 and 9 hours during the night reduced from $40.8 \%$ in 1960 to $23.5 \%$ in 2000-2001 [29], showing that not only has decreased the sleep time of adolescents, but also their sleep time is not suitable for sleeping and they often go to bed too late.

Owens et al. [30] and Spilsbury et al. [31] found that most adolescents often sleep with a great delay, which is consistent with the results of the present study. Finally, it was noteworthy that $69.5 \%$ of the adolescents participating had a severe problem in sleep efficiency on the Pittsburgh Sleep Quality Scale. The findings showed that $2.7 \%$ had severe abnormalities in their daily functioning. In the study by Roberts et al., it was found that $7 \%$ of adolescents had a severe daytime tiredness and disruption and had many problems in their daily functioning, which was lower than the value obtained in the present study. Moreover, in the Roberts's study, it was stressed that a large percentage of adolescents have many problems in their daily life spheres due to disturbances in their sleep cycle that are in line with the findings of the present study [32]. It is worth mentioning that every adolescent needs sleep to maintain physical and psychological balance. It is obvious that an adolescent who has troubles 
in sleeping and waking cycles will have many problems in the daily life process. These groups are often bored, feel tired, and fail in education and occupation. Aggression and significant behavioral and learning problems are evident in them.

In the present study, $6 \%$ of the adolescents had severe sleep disorders. In a study on 4000 adolescents, $14 \%$ of them had one or more symptoms of sleep disorder, and 5\% had complete sleep disorders based on DSMIV diagnostic criteria, which was similar to that found in the present study [33]. In a multinational study in four European countries, including the UK, France, Germany, and Italy, the prevalence of sleep disorder among 1125 adolescents aged 15 to 18 was $25.7 \%$ [9]. The other result of the study was that $16.7 \%$ of the adolescents had sleep disorders and $11.5 \%$ had difficulty in continuing sleep. In the Roberts's study, it was found that $0.06 \%$ of adolescents had a sleep disorder that was lower than that of the present study [32]. In the study by Kaneita et al, $11.3 \%$ of adolescents had sleep disorders, $5.5 \%$ had impaired sleep [11], and $24.7 \%$ had a sleep deprivation feeling during the day. The origin of the disorder at the onset and continuation of sleep and daytime sleepiness in adolescents can be in the psychological and family conditions of the adolescent or early wake-up in the morning to go to school or watching television a lot. Adolescence is associated with quick changes in physical and social development. The presence of the disorder and disturbances in the onset and continuation of sleep and daytime sleepiness, which is associated with problems with the onset and continuation of daytime sleep in adolescents, have a negative impact on physical growth and the social development of this group of adolescents with peers and the community. In the study by Zahang et al, the prevalence of sleepiness was $75.3 \%$ that was more than the value obtained in the present study [15].

The prevalence of daily fatigue in the present study was $31.6 \%$ among adolescents, which is somehow the same value as in the study of Moderates et al that reported a rate of $28.8 \%$
[28]. Moreover, $10.2 \%$ of the adolescents had respiratory disruptions. In Zahang et al. study, sleep disorder value was obtained as $14.5 \%$ [15]. In Amin Tehran et al. study, $11.5 \%$ of the sample in the research group had sleep disorders [14]. The reported value in this study is a little lower than the values reported in the two mentioned studies. In the sample of the present study, $9.4 \%$ of the adolescents had snoring during the night. There has been little research on the prevalence of snoring in adolescents. In the study by Modarresi et al, the prevalence of this disorder in adolescents was $4.7 \%$, which is lower than the value of the present study [28], and $8.5 \%$ of the adolescents experienced nightmares at night. In a study that was conducted in Iran, the prevalence of this sleep disorder was $23.1 \%$ [23]. In the study of Fandano et al., the prevalence of nightmare sleep disorder was $9.3 \%$, which is roughly the same as the value found in the present study [5]. Moreover, 27.8\% of the adolescents had a sleep disorder due to daily activity and $26.3 \%$ of the adolescents had a daily disorder due to improper sleep cycle and poor quality of sleep. In another study, $10.4 \%$ of adolescents were severely weak in their academic performance due to their poor sleep quality and were often late in the class [28], 16.9\% of adolescents had restless leg syndrome, $11.7 \%$ had a circular motion of the limbs, and $11 \%$ had the sleepwalking disorder. In the research by Ram et al, the prevalence of restless leg syndrome disorder was 0.02 [34]. In the Wang's research, in China, the prevalence of these disorders was $9.4 \%$ [12]. In a study by Fredando et al, the prevalence of these three disorders, which are placed in the Parasomnia category was $9.3 \%$ [5], and $18.2 \%$ of adolescents had secondary insomnia. In the study by Roberts et al, the incidence of secondary insomnia was $4.1 \%$; and in another study, the prevalence of this disorder was $23.5 \%$ [32], and $26.4 \%$ of the adolescents participating in the study experienced anxiety symptoms and they were very anxious. Many studies have shown that most patients with sleep disorders experience great anxiety 
and concern. In a study in Iran, which was conducted among adolescents in Isfahan, the prevalence of anxiety and worrying emotions in adolescents with sleep disorders was $24.1 \%$ [28].

\section{Conclusion}

The results of the present study showed that the sleep quality of many adolescents participating in the study was undesirable and sleep disorder was very prevalent. Sleep disorders are of the most common disorders in adolescents and children, and their parents are greatly worried about the sleep disorders of their children. Non-pharmacological treatments are often the recommended treatments by most psychiatrists and healthcare practitioners. There is a great empirical support for these types of treatments for bettering the condition of adolescents with a sleep disorder. However, all physicians and healthcare providers are advised to provide a comprehensive evaluation of adolescents first to obtain an accurate knowledge about the exact cause of the disorder. Evaluation of sleep disorders in adolescents should be done in two psychiatric and medical dimensions. In the next step, appropriate therapeutic approaches associated with them should be proposed. A multi-faceted approach should be considered in the treatment of adolescent sleep disorder. The design and framework for prevention education is very useful for preventing other adolescents from developing sleep disorders. It is hoped that the results of this study are of interest to parents of adolescents, psychiatrists, psychologists, and other mental health care providers and optimal therapeutic programs are designed for improving sleep quality and reducing sleep disorders in adolescents.

\section{Acknowledgments}

Hereby, the authors show their heartfelt gratitude to Gonabad and security officials of this department, the management and the deputy of the second year high schools in Gonabad and all adolescents who participated in the study.

\section{Authors' contributions}

Study design, data collection, analysis, and manuscript preparation: SS

Data analysis: HRT

All authors have read and approved the final version.

\section{Conflict of Interest}

"The authors declared that they have no competing interests".

\section{Funding}

The author (s) received no financial support for the research, authorship and/or publication of this manuscript.

\section{Availability of data and materials}

The datasets used and/or analyzed during this study are available from the corresponding author on reasonable request.

\section{References}

1- Parsaie Rad E, Amir Ali Akbari S, Sadeghniiat Kh, Alavi Majd H. Relationship between sleep disorder and pregnancy depression in primigravidae referring to health-treatment centers of Ahvaz Jundishapur university of medical sciences in 2010. Shahid Sadoughi University of Medical Sciences2011; 19(4): 454-62.

2- Afkham Ebrahimi A, Ghale Bandi MF, Salehi M, Kafian Tafti AR, Vakili Y, Akhlaghi Farsi E. Sleep parameters and the factors affecting the quality of sleep in patients attending selected clinics of Rasoul-eakram hospital. Razi Journal of Medical Sciences2008; 58: $31-8$.

3- Mansouri A, Tavakkol Z, Mohammadi Farrokhran E, Mokhayeri Y, Fotouhi A. Sleep quality of students living in dormitories in Tehran university of medical sciences (TUMS) in 2011. Iranian Journal of Epidemiology2012; 8(2): 71-82.

4- Rev de Castro J, Gallo J, Loureiro H. Tiredness and sleepiness in bus drivers and road accidents in Peru: a quantitative study. Rev Panam Salud Publica2004; 16(1): 11-8

5- Fernando AT, Samaranayake CB, Blank CJ, Roberts G, Arroll B. Sleep disorders among high school students in New Zealand. J Prim Health Care2013; 5(4): 276-82. 6- Ohayon MM, Sagales T. Prevalence of insomnia and sleep characteristics in the general population of Spain. Sleep Med2010; 11(10): 1010-8.

7- Falloon K, Arroll B, Elley CR, Fernando A. The assessment and management of insomnia in primary care. BMJ2011; 342: d2899 
8- Mindell JA, Meltzer LJ. Behavioural sleep disorders in children and adolescents. Ann Acad Med2008; 37(8): $722-8$.

9- Ohayon MM, Roberts RE, Zulley J, Smirne S, Priest RG. Prevalence and patterns of problematic sleep among older adolescents. J Am Acad Child Adolesc Psychiatry2000; 39(12):1549-56.

10- Kaneita Y, Ohida T, Osaki Y, et al. Insomnia among japanese adolescents: a nationwide representative survey. Sleep2006; 29(12): 1543-50.

11- Kang V, Shao J, Zhang K, Mulvey M, Ming X, Wagner GC. Sleep deficiency and sleep health problems in Chinese adolescents. Clin Med Insights Pediatr2012; 6: 11-7.

12- Wang G, Xu G, Liu Z, Lu N, Ma R, Zhang E. Sleep patterns and sleep disturbances among Chinese schoolaged children: Prevalence and associated factors. Sleep Med2013; 14(1): 45-52.

13- Lund HG, Reider BD, Whiting AB, et al. Sleep patterns and predictors of disturbed sleep in a large population of college students. J Adolesc Health2010; 46(2): 124-32.

14- Zhang Z, Chen T, Jin X, Yan Ch, Shen X, Li Sh. Sleep Patterns, Sleep Problems and Associations with Reported Sleep Quality in Chinese School-Aged Children. Am J Public Health Res2013; 1(4): 93-100.

15- Amintehran E, Ghalehbaghi B, Asghari A, Jalilolghadr Sh, Ahmadvand A, Foroughi F. High prevalence of sleep problems in school- and preschool-aged children in Tehran: a population based study. Iran J Pediatr2013; 23: 45-52.

16- Wolfson AR. Adolescents and emerging adults' sleep patterns: new developments. J Adolesc Health2010; 46(2): 97-9.

17- Wong MM, Brower KJ, Nigg JT, Zucker RA. Childhood sleep problems, response inhibition, and alcohol and drug outcomes in adolescence and young adulthood. Alcohol Clin Exp Res2010; 34(6): 1033-44.

18- Lewandowski A, Ward T, Palermo T. Sleep problems in children and adolescents with common medical conditions. Pediatr Clin North Am2011; 58(3): 699-713.

19- Daniel WW. A foundation for analysis in the health sciences. Biostatistics. New York: Wiley; 1991.

20- Razaziyan N, Najafi F, Mahdavi P, Aghaei A. Prevalence of sleep disorders in patients with multiple sclerosis. Journal of Mazandaran University of Medical Sciences2014; 23(110): 175-81.
21- Rahimian Boogar I, Ghodrati Mirkouhi M. Role of workload, sleep, mental health and individual factors in occurrence of nursing errors. Journal of Gorgan University of Medical Sciences2013; 15(3): 103-9.

22- Russo PM, Bruni O, Lucidi F, Ferri R, Violani C. Sleep habits and circadian preference in Italian children and adolescents. $J$ Sleep Res2007; 16(2): 163-9.

23-Johnson EO, Breslau N. Sleep problems and substance use in adolescence. Drug Alcohol Depend2001: 64: 1-7.

24- Short MA, Gradisar M, Lack LC, Wright HR, Dohnt

H. The sleep patterns and well-being of Australian adolescents. $J$ Adolesc2013; 36(1): 103-10.

25- Cornelius LJ. Health habits of school-age children. $J$ Health Care Poor Underserved1991; 2(3): 374-95.

26- Meltzer LJ, Johnson C, Crosette J, Ramos M, Mindell

JA. Prevalence of diagnosed sleep disorders in pediatric primary care practices. Pediatrics2010; 125(6): 1410-8.

27- Mousavi F, Golestan B, Matini E, Tabatabaei

R. Sleep quality and related factors in interns and externs of Tehran Islamic Azad University medical students. Medical Sciences Journal of Islamic Azad University, Tehran Medical Branch2011; 20(4): 278-84.

28- Modarresi MR, Faghihinia J, Akbari M, Rashti A. The relation between sleep disorders and academic performance in secondary school students. Isfahan University of Medical Sciences2012; 30(206): 1456-67. 29- Nyberg G, Ekelund U, Marcus C. Physical activity in children measured by accelerometry: stability over time. Scand J Med Sci Sports2009; 19(1): 30-5.

30- Owens JA, Spirito A, McGuinn M, Nobile C. Sleep habits and sleep disturbance in elementary school-aged children. J Dev Behav Pediatr2000; 21(1): 27-36.

31- Spilsbury JC, Storfer-Isser A, Drotar D, et al. Sleep behavior in an urban US sample of school-aged children. Arch Pediatr Adolesc Med2004; 158(10): 988-94.

32- Roberts RE, Roberts CR, Chan W. Ethnic differences in symptoms of insomnia among adolescents. Sleep 2006; 29(3): 359-65.

33- Roberts RE, Roberts CR, Chan W. Persistence and change in symptoms of insomnia in adolescents. Sleep2008; 31(2): 177-84.

34- Ram S, Seirawan H, Kumar SK, Clark GT. Prevalence and impact of sleep disorders and sleep habits in the United States. Sleep Breath2010; 14(1): 63-70.

\footnotetext{
Copyright $(\mathrm{C}) 2016$ ASP Ins. This open-access article is published under the terms of the Creative Commons Attribution-NonCommercial 4.0 International License which permits Share (copy and redistribute the material in any medium or format) and Adapt (remix, transform, and build upon the material) under the Attribution-NonCommercial terms.
} 